\title{
Extending the Meta-design Theory: Engaging Participants as Active Contributors in Virtual Worlds
}

\author{
Benjamin Koehne, David Redmiles, and Gerhard Fischer \\ Donald Bren School of Information and Computer Sciences, \\ University of California at Irvine, 6210 Donald Bren Hall \\ Irvine, CA 92697-3425 USA \\ \{bkoehne, redmiles\}@ics.uci.edu, gerhard@colorado.edu
}

\begin{abstract}
Meta-design has emerged as a theoretical framework by supporting open systems that allow end-users to become designers in dynamic use contexts. Our research is grounded in the objectives that (1) the meta-design theory can inform the design of virtual worlds, and that, in return, (2) observations and insights from virtual worlds can broaden the meta-design theory. Our work is based on 80 hours participant observation and additional interviews.
\end{abstract}

Keywords: Meta-design, virtual worlds, end-user design, human-centered design, science of design, open evolvable systems, empowerment of the user.

\section{Introduction}

In recent years, persistent virtual 3D-environments, in the following referred to as 'virtual worlds (VW)', have drawn increasing interest both from industry and academia $[1,2,3]$. VWs provide persistent virtual environments in which users can interact by controlling a virtual avatar. The avatar can move in the VW and interact with other avatars and virtual artifacts. Two of the most successful and widely used applications of VWs are:

- massively-multiplayer online role-playing games (MMORPGs), such as 'Lord of the Rings Online' (LOTRO), where millions of players worldwide come together and spend considerable amounts of time in order to engage in collaborative tasks or simply to engage in social interactions; and

- open-ended VWs, such as Second Life ${ }^{l}$ and OpenSimulator $^{2}$, where users engage in many endeavors paralleling and augmenting daily life and work, including advanced, simulated environments that support various research efforts (e.g. Center for Computer Games \& Virtual Worlds at UC Irvine ${ }^{3}$ ).

\footnotetext{
${ }^{1}$ See: www.secondlife.com

${ }^{2}$ See: www.opensimulator.org

${ }^{3}$ See: http://cgvw.ics.uci.edu
} 
In this paper we re-examine and evolve the current theories of meta-design based on observations made within VWs. We present accounts of both a gaming-oriented and an open-ended VW system. We juxtapose our observations in the MMORPG 'Lord of the Rings Online' (LOTRO) and the open-ended environment of Second Life (SL). A series of interviews with individual players of LOTRO and users of SL provide individual viewpoints and contextualize the ethnographic investigation.

\section{Virtual World Systems: Second Life and LOTRO}

SL represents an open-ended VW system that allows users to modify and extend the virtual environment with high flexibility and in great detail. Users build up virtual artifacts from simple building blocks and can customize appearance and behavior of these objects. SL does not prescribe a common goal for its users. Instead users explore the possibilities of the environment on their own accord.

LOTRO attracts users with a complex storyline and visually appealing graphics. Users of LOTRO can engage in various activities that ultimately lead to the development of virtual characters within the given regulations of the game framework. The creation of virtual artifacts in LOTRO is tied to the game logic and more constrained compared to open-ended VWs such as SL.

We see a dual relationship between VWs and concepts of meta-design that further motivates our argument to consider VWs for the extension of meta-design theory in general. In this discussion we specifically focus on user participation. Based on existing meta-design concepts, VWs can employ scaffolding processes that guide casual users. Similarly, the unique characteristics of popular VWs and the users they attract can inform the extension of user participation theory.
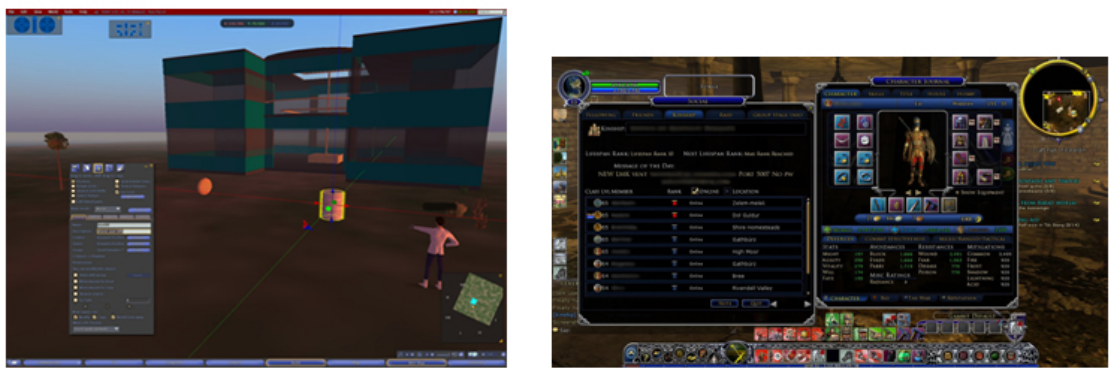

Fig. 1. Second Life (left) and LOTRO (right)

VWs have great potential to affect meta-design theory by providing environments that enable casual users to, possibly unintentionally, engage in meta-design practice.

\section{Methodology}

Our investigation of LOTRO and SL to re-examine and evolve the current theories of meta-design is focused on the following research objectives: 
- develop additional examples of meta-design for worlds that have no laws and boundaries;

- support the empowerment of end-users that are not initially interested or motivated to conduct design practice;

- assess the duality between VWs and meta-design, i.e.: how does meta-design affects practices in VWs and vice versa; and,

- analyze the support for meta-design in both unique environments, focusing on the benefits and shortcomings of the gaming-oriented and the open-ended environment under study.

Our methodology draws from virtual ethnography $[6,10]$ and from multi-sited ethnography [9], which allows us to supplement our findings with interviews with users outside of the VW context.

We conducted 80 hours of participant observation in LOTRO and SL. We took part in various everyday activities in both VWs with other users. Additionally, we conducted 6 semi-structured interviews with players of LOTRO and two interviews with designers in SL. Each interview lasted between 40 and 60 minutes.

The long-term participant observation allowed us to learn about individual activities of the users in the VWs. The semi-structured interviews provided us with personal perspectives and reflections. We recruited informants for the interviews through snowball-sampling in the player community of LOTRO and SL. The interviews focused on themes of the VWs that we related to meta-design activities based on our previous participant observation. Additionally, we asked the participants about their individual personal backgrounds and their general motivations to participate in the VWs.

Patterns and themes based on our observations were analyzed using open-coding techniques [8]. The meta-design concepts identified in the following chapter were used to filter and code the collected data. The data analysis focused on practices framed by meta-design in the VWs. We were specifically interested in the changing roles during different stages of participation. We structure our findings based on central concepts of meta-design originally formulated in [5].

\section{Discussion: Meta-design Concepts in SL and LOTRO}

SL and LOTRO represent two systems that implement elements of meta-design in quite different ways. Both systems can individually serve as systems for exemplifying various concepts of meta-design theory. A synthesis of the exemplified concepts allows for a more detailed analysis of VW system features that particularly affect casual end-user participation. Table 1 provides a comparison of the central meta-design concepts and the corresponding properties of the VW systems included in the analysis. An expanded discussion of these concepts is available in [7]. 
Table 1. Meta-Design Concepts in Virtual Worlds

\begin{tabular}{|c|c|c|}
\hline Meta-Design Concept & LOTRO & Second Life \\
\hline convivial tools & $\begin{array}{l}\text { Adventure \& } \\
\text { leveling system }\end{array}$ & prim design tools \\
\hline domain orientation & $\begin{array}{l}\text { goals from fixed set } \\
\text { in the fantasy world } \\
\text { context }\end{array}$ & $\begin{array}{l}\text { user-imagined } \\
\text { goals, open- } \\
\text { endedness }\end{array}$ \\
\hline $\begin{array}{l}\text { open, evolvable } \\
\text { systems }\end{array}$ & $\begin{array}{l}\text { limited } \\
\text { customization }\end{array}$ & $\begin{array}{l}\text { unrestricted } \\
\text { customization }\end{array}$ \\
\hline $\begin{array}{l}\text { underdesigned } \\
\text { systems }\end{array}$ & $\begin{array}{l}\text { fixed fantasy world } \\
\text { context }\end{array}$ & $\begin{array}{l}\text { minimalist } \\
\text { environment }\end{array}$ \\
\hline $\begin{array}{l}\text { collaborative work } \\
\text { practices }\end{array}$ & $\begin{array}{l}\text { high cooperation } \\
\text { amongst players }\end{array}$ & $\begin{array}{l}\text { limited cooperation, } \\
\text { specialization }\end{array}$ \\
\hline
\end{tabular}

VWs, in particular MMORPGs, draw a large number of users. As our analysis has shown, users join the environments with very different aspirations. VWs can accommodate a very diverse user base by providing a system of technological and social structures that is sufficiently flexible to provide individuals with discrete virtual spaces within the larger system. Current systems discussed in the context of metadesign mostly lack opportunities to unpack rich ecologies of participation. Our observations in the VWs point to a collaboration practices as deciding factors to support different levels of participation in the game- and open-ended context.

LOTRO makes casual gamers gradually aware of the functional and social properties of the game. The scaffolding system in LOTRO is based on elements in the VW providing guidance and works in close connection with collaboration between the players. While SL represents an open environment with great freedom of creative interaction, LOTRO's strength lies in the integration of collaborative community effects.

VWs offer an opportunity to study the effects of collaboration on the way casual users move through ecologies of participation. Technical scaffolding systems alone are not sufficient. Instead, social community components need to make collaboration tools more accessible and attractive for casual users.

Collaboration in VWs is closely tied to the social structure of the system. In LOTRO, collaboration is initially triggered by the functional structure of the game that creates the need for players to find collaborators for difficult adventures or to create powerful artifacts. Over time, the recurring need for collaborative activities creates social networks amongst players that, once initially established, are sustained for a longer time period. In this eco-system of social collaboration, the motivation to help others becomes detached from traditional reward systems. Respect and a good reputation become equally or more important than monetary rewards or other character improvements.

These examples show that a useful extension of the meta-design framework would be a careful analysis of user roles on an individual level. Intrinsic motivations to engage in creative activities only come forward in large scale systems such as LOTRO or SL. 
Users of VWs can dynamically switch between the roles of passive consumers and active contributors. While the degree of contributions differs between LOTRO and $\mathrm{SL}$, it is common in both worlds that users are not locked into either the designer or the user role.

The domain-oriented gaming environment creates opportunities for need-based attendance to creative practices. Players can also decide to completely neglect crafting activities. The open-ended environment of SL allows users to switch between observer and designer roles. However, the complexity imposed by the available design tools creates a higher entry barrier to become a designer. Meta-designers need to find a middle ground between complex and universal design tools as exemplified in SL and socially embedded creative activity as exemplified in LOTRO. Our results point to a priority for socio-technical contexts that allow users to develop their roles based on social interactions through collaborative activities with other users. More experienced users develop their own strategies while novice users require guidance to be gradually introduced to the system functionalities. There is not a perfect solution for this trade-off problem.

However, our analysis of two distinct VWs leads us to suggest an extension of the seeding, evolutionary growth and reseeding model (SER) [4] that clearly focuses on support during transition phases between different stages of participation. In the VWs other users constantly provide seeds for design practice. The leveling system in LOTRO gradually prepares players with coordinated stages and sub-goals on their progression from novice players to experienced performers. Combined with the visibility of fine-grained design processes observed in SL, our findings suggest a combination of these two concepts. Designers and design artifacts undergo stages of evolutionary growth. Design products are re-introduced in the environments and serve users on different levels of participation as examples for novel design projects.

Meta-design theory can benefit designers of VWs. The meta-design concepts discussed in the qualitative investigation can guide game designers but also designers of open-ended systems. Possible design recommendations can be imagined by combining the social community elements found in LOTRO and the accessible design tools in SL. Studying VWs can contribute to broadening the theory of meta-design. VWs inherit qualities that can inform an expansion of the theory by providing additional systems to exemplify meta-design concepts. The large numbers of casual users with diverse interests in our view represent the missing masses that meta-designers should focus on. Gaming-oriented VWs employ concepts that gradually empower end-users while keeping them motivated and engaged. Social structures create an environment that fosters cooperation amongst the players.

Meta-designers look for tools to empower end-users to tailor systems towards their needs. Open-ended environments like SL provide these types of tools in virtual spaces. The tools do not discriminate between novice users and users with design experience in different domains. Studying tools that create an even, flat entry level for all users can inform concepts of meta-design tools in general.

Based on our study, we have identified a rich duality between VWs and metadesign. Meta-design potentially provides VW designers with a useful analytic design for user participation and open systems. VWs extend meta-design theory by providing a class of new systems to exemplify meta-design concepts. 
Initially, it appears simple to dismiss LOTRO as a system for meta-design practices based on the regulations imposed on the users and the limited design opportunities in the VW. However, our analysis has brought to light concepts that metadesigners are well-advised to take into account.

\section{Conclusion and Future Work}

This work opens many avenues for future research. First, some open-ended VWs such as SL and, more particularly, the open source counterpart, OpenSimulator, provide means for extension through source code modification. Extending them to provide scaffolding that makes meta-design more explicit in the VWs that players or end users experience is one real possibility. Second, the potential that meta-design holds for empowering end users is an on-going exploration. The implications in altering the power between the roles of end users and designers are just being understood.

Acknowledgments. This work was supported by the U.S. National Science Foundation under grant \#0808783. We would like to thank our colleagues at UC Irvine and the University of Colorado at Boulder for their insightful comments.

\section{References}

1. Bainbridge, W.S.: The Scientific Research Potential of Virtual Worlds. Science 317(5837), 472-476 (2007)

2. Costabile, M.F., Fogli, D., Mussio, P., Piccinno, A.: A meta-design approach to end-user development. Paper Presented at the 2005 IEEE Symposium on Visual Languages and Human-Centric Computing (2005)

3. Fischer, G.: End-User Development and Meta-design: Foundations for Cultures of Participation. In: End-User Development, pp. 3-14 (2009)

4. Fischer, G., Grudin, J., McCall, R., Ostwald, J., Redmiles, D., Reeves, B., Shipman, F.: Seeding, evolutionary growth and reseeding: The incremental development of collaborative design environments. In: Olson, G.M., Malone, T.W., Smith, J.B. (eds.) Coordination Theory and Collaboration Technology, pp. 447-472. Lawrence Erlbaum, Mahwah (2001)

5. Fischer, G., Scharff, E.: Meta-design: design for designers. In: Proceedings of the 3rd Conference on Designing Interactive Systems: Processes, Practices, Methods, and Techniques. ACM, New York (2000)

6. Hine, C.: Virtual ethnography. SAGE, London (2000)

7. Koehne, B., Redmiles, D., Fischer, G.: Details on Extending the Meta-Design Theory: Results from Participant Observation of Active Contributors in Virtual Worlds (2011), http://www.isr.uci.edu/tech_reports/UCI-ISR-11-1.pdf (March 14, 2011)

8. Lofland, J.: Analyzing social settings: a guide to qualitative observation and analysis, 4th edn. Wadsworth/Thomson Learning, Belmont, CA (2006)

9. Marcus, G.E.: Ethnography in/of the World System: The Emergence of Multi-Sited Ethnography. Annual Review of Anthropology 24, 95-117 (1995)

10. Nardi, B.A.: My life as a night elf priest: an anthropological account of world of warcraft. The University of Michigan Press, Ann Arbor (2010) 